\title{
Discrete Arrow-Pratt indexes for risk and uncertainty
}

\section{Aurélien Baillon $^{1}$ D $\cdot$ Olivier L'Haridon ${ }^{2}$}

Received: 5 December 2019 / Accepted: 23 September 2020 / Published online: 7 October 2020 (c) The Author(s) 2020

\begin{abstract}
The Arrow-Pratt index, a gold standard in studies of risk attitudes, is not directly observable from choice data. Existing methods to measure it rely on parametric assumptions. We introduce a discrete Arrow-Pratt index, and its relative counterpart, that can be directly obtained from choices. Our approach is general: it is (i) non-parametric, (ii) applicable to both risk and uncertainty, (iii) and robust to probability transformation, non-additive beliefs and multiple priors. Our index can also be used to characterize various decision models through various simple consistency requirements. We analyze its properties and demonstrate how it can be measured.
\end{abstract}

Keywords Risk aversion - Arrow-Pratt index · CARA - CRRA · Preference foundation

\section{JEL Classification C91 · D81}

Arrow (1965) and Pratt (1964) proposed ${ }^{1}$ to measure a decision maker's degree of risk aversion by the index $A(x) \equiv-\frac{u^{\prime \prime}(x)}{u^{\prime}(x)}$. Under expected utility, the degree of concavity of utility determines the risk premium for a given risk. If the decision maker faces an infinitesimal and actuarially fair risk $\varepsilon$ with variance $\sigma_{\varepsilon}^{2}$, the risk premium for $\varepsilon$ is approximately $\frac{\sigma_{\varepsilon}^{2}}{2} \times A$ (Pratt 1964). If the Arrow-Pratt index $A$ is constant, an increase in the decision maker's wealth leaves the risk premia unaffected. Similarly, if the relative Arrow-Pratt index $R(x) \equiv x A(x)$ is constant, multiplying the initial wealth and the risk by a common factor does not influence the relative risk premia. The

Electronic supplementary material The online version of this article (https://doi.org/10.1007/s00199020-01315-8) contains supplementary material, which is available to authorized users.

$\bowtie$ Aurélien Baillon

baillon@ese.eur.nl

1 Erasmus School of Economics, Erasmus University Rotterdam, P.O. Box 1738, 3000 DR Rotterdam, The Netherlands

2 Department of Economics, Université de Rennes 1, Univ Rennes, CREM UMR 6211, 7, place Hoche, 35000 Rennes, France

1 They were actually preceded by de Finetti (1952). See Montesano (2009) for a discussion. 
Arrow-Pratt index can be viewed as the gold standard for the study of risk attitudes (Machina 1982) under expected utility. Countless are the papers that make use of it.

Surprisingly, a fundamental issue of the Arrow-Pratt index has never been resolved: it is not directly observable from choices. First, it uses utility as input, which is not directly observable. Second, it is based on (second) derivatives and, therefore, on infinitesimal changes, which can at best be approximated using complex data. The latter issue is not peculiar to the Arrow-Pratt index. It similarly applies to elasticities, for instance, such as the price elasticity of demand. A pragmatic solution is to use discrete approximations, i.e. an arc elasticity.

Parametric approaches have been widely used to estimate Arrow-Pratt indexes. Fitting smooth utility functions allows researchers to compute derivatives, but the choice of the parametric functions constrains the values of the derivatives. For instance, fitting an exponential function forces the inferred Arrow-Pratt index to be insensitive to changes of wealth. Furthermore, a parametric method developed for one family is not guaranteed to work for another family (see Sect. 3).

This paper introduces a discrete approximation (not relying on infinitesimal changes) of the Arrow-Pratt index that is obtained from two indifferences (i.e., directly from choices). ${ }^{2}$ Our approach is general in three ways. First, it is non-parametric, and can, therefore, be applied without having to assume, ex ante, a functional form. Second, it can be used both if probabilities are known (risk) and unknown (uncertainty). Third, we propose a version that is robust to common violations of expected utility under risk and uncertainty.

Our discrete Arrow-Pratt indexes are derived from (endogenous) preference midpoints. The preference midpoint $m$ between outcomes $x$ and $y$ (with $x<y$ ) is the outcome such that substituting $x$ for $m$ is worth the same (in a sense specified later) as substituting $m$ for $y$. Under expected utility, it implies that the utility $u$ of $m$ is half-way between the utility of $x$ and the utility of $y$. This is also true for biseparable utility, a more general model of decision under risk and uncertainty. Preference midpoints are commonly used in the theoretical literature, to define axioms and properties (Vind 1991; Ghirardato et al. 2003; Köbberling and Wakker 2003; Abdellaoui et al. 2007; Baillon et al. 2012; Dean and Ortoleva 2017; Werner and Zank 2019; Ghirardato and Pennesi 2019). The more concave the utility is, the closer the preference midpoint is from the lower wealth $x$ (Baillon et al. 2012). Comparing the distances $m-x$ to $y-m$ provides, therefore, a simple way to study utility curvature. We define the discrete Arrow-Pratt index of the interval $[x, y]$ as the difference between the inverse of these distances:

$$
\mathcal{A}_{[x, y]}=\frac{1}{m-x}-\frac{1}{y-m}
$$

Our formula naturally arises from linear approximations of the derivatives of $u$. Its slope between $x$ and $y$ is $\mathcal{S}_{[x, y]}=\frac{u(y)-u(x)}{y-x}$. It is the average marginal utility $u^{\prime}$ over $[x, y]$. To study concavity, we can consider the slope of the slope, on the left and on

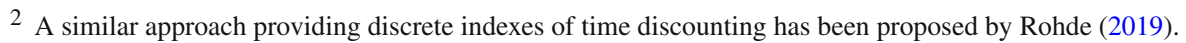


the right of the preference midpoint. ${ }^{3}$ We define the slope of the slope on $[x, y]$ as:

$$
\mathcal{C}_{[x, y]}=\frac{\mathcal{S}_{[m, y]}-\mathcal{S}_{[x, m]}}{\frac{1}{2}(y-x)} .
$$

If the slope is constant, then $\mathcal{C}$ is 0 . The denominator normalizes $\mathcal{C}$ such that $\mathcal{C}=u^{\prime \prime}$ whenever $u^{\prime \prime}$ is constant (as $\mathcal{S}=u^{\prime}$ whenever $u^{\prime}$ is constant). ${ }^{4}$ Note that, $m$ being a preference midpoint, $u(y)-u(m)=u(m)-u(x)=\frac{u(y)-u(x)}{2}$. Hence, Eq. 2 can be rewritten as:

$$
\mathcal{C}_{[x, y]}=\left(\frac{1}{y-m}-\frac{1}{m-x}\right)\left(\frac{u(y)-u(x)}{y-x}\right) .
$$

The Arrow-Pratt index, dividing $u^{\prime \prime}$ by $u^{\prime}$, remains constant for any affine transformation of $u$. To obtain the same property, we can divide $\mathcal{C}$ by $\mathcal{S}$.

$$
\mathcal{A}_{[x, y]}=-\frac{\mathcal{C}_{[x, y]}}{\mathcal{S}_{[x, y]}}=\frac{1}{m-x}-\frac{1}{y-m} .
$$

Rohde (2019) preceded us in using preference conditions to build a discrete approximation of a continuous index. Rohde showed how to transform time tradeoffs into an index of decreasing impatience, approximating that of Prelec (2004). Her approach, however, is not based on preference midpoints.

The first section of the paper provides the formal definition of the discrete ArrowPratt index. Requiring stronger or weaker consistency of the index characterizes (i.e. axiomatizes) several models of decision under risk and uncertainty. The consistency requirement generalizes existing axiomatizations of expected utility for binary state spaces. Section 2 shows that the discrete indexes preserve the properties of the original ones, $A(x)$ and $R(x)$. It implies that common assumptions in economics and finance such as decreasing absolute risk aversion or increasing relative risk aversion become directly testable with our discrete indexes. Section 3 discusses elicitation of the indexes and alternatives and the Electronic Supplementary Material demonstrates practical feasibility. Section 4 concludes.

\section{Preference foundation}

\subsection{Diminishing rates of substitution}

Consider the indifference curves on Fig. 1a, in which the horizontal axis describes the quantity of good 1 and the vertical axis that of good 2. The (discrete) rate of substitution of good 1 for good 2 (represented by the cord) is $\frac{r_{1}-r_{2}}{m-x}$ on the first indifference curve

\footnotetext{
3 Physical midpoints $\frac{x+y}{2}$ would not simplify Eqs. 3 and 4 the way preference midpoints do.

${ }^{4}$ Assume $u^{\prime \prime}=a$, and therefore $u(x)=\frac{a}{2} x^{2}+b x+c$ for some $b$ and $c$. Then $\mathcal{S}_{[x, y]}=\frac{\frac{a}{2} y^{2}+b y-\frac{a}{2} x^{2}-b x}{y-x}=$ $\frac{a}{2}(y+x)+b$. Hence, $\mathcal{S}_{[m, y]}-\mathcal{S}_{[x, m]}=\frac{a(y-x)}{2}$ and $\mathcal{C}_{[x, y]}=a$.
} 


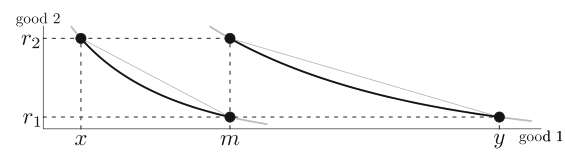

(a) diminishing rates of substitution

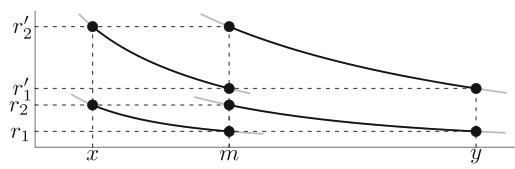

(b) expected utility

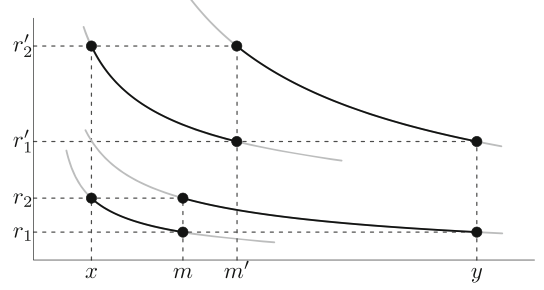

(c) general

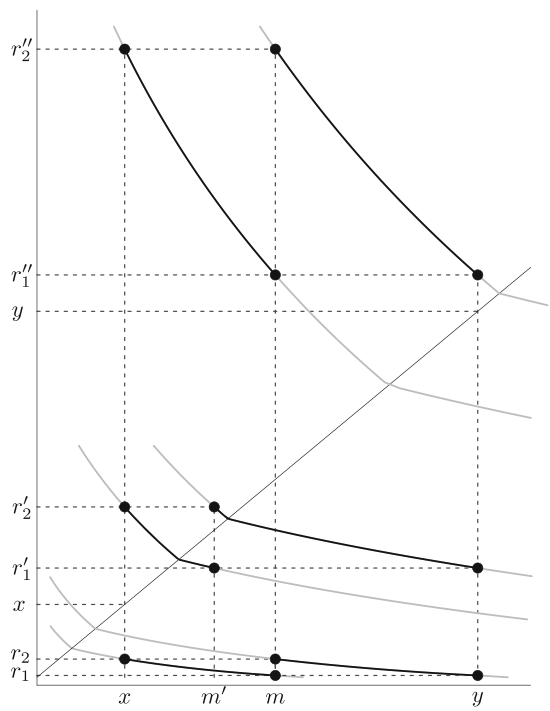

(d) biseparable utility

Fig. 1 Preference midpoints and $\mathcal{A}$-consistency. Note: Biseparable utility is defined by Eq. 7 below

and it diminishes to $\frac{r_{1}-r_{2}}{y-m}$ on the second indifference curve. A larger quantity of good 1 is needed to offset one unit of good 2. The change of rates of substitution is $\left(r_{1}-r_{2}\right)\left(\frac{1}{m-x}-\frac{1}{y-m}\right)$. The second factor of the product captures how much the rate of substitution changes per unit of good 2. Baillon et al. (2012) used such a setting, in which the change of good 2 is kept constant across indifference curves, and interpreted diminishing rates of substitution to mean $m$ being closer to $x$ than to $y$. It implies that $\frac{1}{m-x}-\frac{1}{y-m}$ is positive for diminishing rates of substitution. The same reasoning can be used if we replace goods by state-dependent outcomes, as we do from now on.

The state space $S=\left\{s_{1}, s_{2}\right\}$ consists of two mutually exclusive and exhaustive states of nature. The states may have an objective probability that everyone agrees upon, which would represent the special case of decision under risk. Otherwise, the setting models decision under uncertainty. This general approach allows us to remain agnostic about how people deal with probabilities, whether they are objective, subjective or even multiple as in a set of priors. The outcome set is $\mathbb{R}_{+}$. Bet $(x, y)$ yields outcome $x$ if state $s_{1}$ obtains and $y$ otherwise. The decision maker has preferences $\succsim$ over the set of all bets $\mathbb{R}_{+}^{2}$. Preferences may satisfy:

weak ordering $\succsim$ is complete and transitive;

monotonicity $x_{1} \geq y_{1}$ and $x_{2} \geq y_{2}$ with one of the two inequalities strict imply $\left(x_{1}, x_{2}\right) \succ\left(y_{1}, y_{2}\right)$; and

continuity $\left\{\left(x_{1}, x_{2}\right):\left(x_{1}, x_{2}\right) \succsim\left(y_{1}, y_{2}\right)\right\}$ and $\left\{\left(x_{1}, x_{2}\right):\left(x_{1}, x_{2}\right) \precsim\left(y_{1}, y_{2}\right)\right\}$ are closed subsets of $\mathbb{R}_{+}^{2}$ for each $\left(y_{1}, y_{2}\right)$. 
Structural Assumption 1 Preferences $\succsim$ are defined over $\mathbb{R}_{+}^{2}$ and satisfy weak ordering, monotonicity, and continuity.

Figure 1a displays indifferences $\left(x, r_{2}\right) \sim\left(m, r_{1}\right)$ and $\left(m, r_{2}\right) \sim\left(y, r_{1}\right)$ but the question arises which state should be used as $\mathrm{x}$-axis and which state as $\mathrm{y}$-axis. In the equation below, we allow for both possibilities-state $s_{1}$ as X-axis or state $s_{2}$ as X-axis. The reason will become clear in the next subsection. Thus, we consider:

$$
\left\{\begin{array}{l}
{\left[\left(x, r_{2}\right) \sim\left(m, r_{1}\right) \text { and }\left(m, r_{2}\right) \sim\left(y, r_{1}\right)\right]} \\
\text { or } \\
{\left[\left(r_{2}, x\right) \sim\left(r_{1}, m\right) \text { and }\left(r_{2}, m\right) \sim\left(r_{1}, y\right)\right] .}
\end{array}\right.
$$

Definition 1 We call $m$ a preference midpoint of $[x, y]$ if Eq. 5 holds for some $r_{1}, r_{2}, x, y$, and $m$ with $x<y$.

We now define the index.

Definition 2 We call

$$
\mathcal{A}_{[x, y]}=\frac{1}{m-x}-\frac{1}{y-m}
$$

a discrete Arrow-Pratt index of $[x, y]$ if $m$ is a preference midpoint of $[x, y]$.

It can be shown that under Structural Assumption 1 there always exists a discrete Arrow-Pratt index for some intervals (see Lemma 4 in Appendix B). For general preferences, there may be many discrete Arrow-Pratt indexs of $[x, y]$. The definition is most useful if there is only one such, and this case is analyzed in the next subsection.

\subsection{Consistency of the discrete Arrow-Pratt index}

The most famous model for decision under uncertainty is (subjective) expected utility, according to which there exist a continuous and strictly increasing $u$ on $\mathbb{R}_{+}$and a (subjective) probability $p_{1}$ satisfying $0<p_{1}<1$ such that preferences maximize $\left(x_{1}, x_{2}\right) \rightarrow p_{1} u\left(x_{1}\right)+\left(1-p_{1}\right) u\left(x_{2}\right)$. Then $u$ is unique up to an affine transformation. If probability $p_{1}$ is objective, we say that decision under risk holds. Baillon et al. (2012) showed:

Lemma 1 Under expected utility, if $m$ is a preference midpoint of $[x, y]$, then $u(m)=$ $\frac{u(x)+u(y)}{2}$.

Under expected utility, $m$ is therefore (i) unique for each pair of outcomes $x, y$, and consequently, (ii) independent of $r_{1}$ and $r_{2}$ (as illustrated on Fig. 1a), and (iii) independent of the state to which the outcomes are assigned (justifying the two parts of Eq. 5). Thus, under expected utility, different measurements of $m$, using different $r_{1}, r_{2}$ or different states, always give the same value $m$ and discrete Arrow-Pratt index 
and, in this sense, are consistent. Expected utility ensures consistency of $m$ and thereby, of the discrete Arrow-Pratt index.

Figure 1c depicts a violation of the aforementioned consistency. The discrete Arrow-Pratt index obtained between $x$ and $y$ using $r_{1}$ and $r_{2}$ in state $s_{2}$ is $\frac{1}{m-x}-\frac{1}{y-m}$ but it decreases when using $r_{1}^{\prime}$ and $r_{2}^{\prime}$ (because the preference midpoint $m^{\prime}$ is higher than $m$ ). It creates an inconsistency, with two discrete Arrow-Pratt indexes for one interval $[x, y]$.

Definition $3 \mathcal{A}$-consistency holds if, for each $(x, y) \in \mathbb{R}_{+}^{2}$ with $x<y$, there is at most one $\mathcal{A}_{[x, y]}$.

$\mathcal{A}$-consistency is equivalent to requiring that there is at most one preference midpoints for each interval. ${ }^{5}$ Lemma 1 showed that $\mathcal{A}$-consistency is ensured by expected utility. Theorem 1 shows that the condition is also sufficient to imply expected utility, as an alternative to Savage's (1954) axiomatization for a binary state space. Our axiom is weaker than the tradeoff consistency of Köbberling and Wakker (2003, Corollary 10), and in this sense we generalize their result for two states (see Appendix B). Using Köbberling and Wakker (2003, Section 7), we thus also generalize the result of Gul et al. (1992). All proofs are in Appendix B.

Theorem 1 Under Structural Assumption 1, A-consistency is equivalent to expected utility.

The main goal of this paper is to make the Arrow-Pratt index observable in empirical applications but for empirical, descriptive purposes, one may prefer not to fully rely on expected utility. Since Allais (1953) and Ellsberg (1961), it has been known that people are likely to deviate from expected utility. Models have been proposed to account for these deviations. In our two-state setting, many non-expected utility models actually agree on their representation, i.e., they have the same functional form: biseparable utility (Luce 1991; Ghirardato and Marinacci 2001). Biseparable utility includes (subjective) expected utility, rank-dependent utility (Quiggin 1981), prospect theory for gains (Tversky and Kahneman 1992), maxmin expected utility (Gilboa and Schmeidler 1989), alpha-maxmin expected utility (Ghirardato et al. 2004), and Choquet expected utility (Schmeidler 1989) as special cases.

Under biseparable utility, there exists a continuous and strictly increasing $u$ and decision weights $\pi_{1}, \pi_{2}$ that are strictly between 0 and 1 such that preferences maximize

$$
\begin{cases}\pi_{1} u\left(x_{1}\right)+\left(1-\pi_{1}\right) u\left(x_{2}\right), & \text { if } x_{1}>x_{2} \\ \left(1-\pi_{2}\right) u\left(x_{1}\right)+\pi_{2} u\left(x_{2}\right), & \text { otherwise. }\end{cases}
$$

As for expected utility, $u$ is unique up to an affine transformation. Expected utility is the special case $\pi_{1}=1-\pi_{2}$. Under rank-dependent utility and prospect theory for gains, $\pi_{i}=w\left(p_{i}\right)$ with $p_{i}$ the objective probability of state $s_{i}$. Under prospect theory

\footnotetext{
5 In Appendix B.1, we show how this definition can be reformulated in a series of three indifferences implying a fourth .
} 
for uncertainty, $\pi_{i}$ is the decision weight of state $s_{i}$ and may (but does not have to) be decomposed into $w\left(p_{i}\right)$, with $p_{i}$ the subjective probability of state $s_{i}$. Under maxmin expected utility, the decision maker has a set of priors about the probability of states $s_{1}$ and $s_{2}$ and $\pi_{i}$ is the infimum of the set of priors for state $s_{i}$. Under alpha-maxmin expected utility, $\pi_{i}$ is a linear combination of the infimum and the supremum of the set of priors for state $s_{i}$.

As can be seen in Eq. 7, biseparable utility consists of two expected-utility parts. For a given bet, which part is used depends on which state receives the highest outcome. It is therefore not surprising that indifference curves for biseparable utility have a kink at the identity line, where the state-ordering of the outcomes changes (see Fig. 1d). Using $r_{1}$ and $r_{2}$ with $r_{1}, r_{2}<x \leq y$ in state $s_{2}$ (i.e., below the identity line) gives $\mathcal{A}_{[x, y]}=m-x-\frac{1}{y-m}$. Using $r_{1}^{\prime \prime}$ and $r_{2}^{\prime \prime}$ with $r_{1}^{\prime \prime}, r_{2}^{\prime \prime}>y \geq x$ (i.e., above the identity line) yields the same. Hence the discrete Arrow-Pratt index is consistent when we measure it below or above the identity line. However, we do observe an inconsistency if one uses indifferences "crossing" the identity line, e.g., $r_{1}^{\prime}$ and $r_{2}^{\prime}$ such that $x<r_{1}^{\prime}<r_{2}^{\prime}<y$ on Fig. 1d. In that case, $\mathcal{A}_{[x, y]}=\frac{1}{m^{\prime}-x}-\frac{1}{y-m^{\prime}}$, which means it is not unique for a given interval $[x, y]$. We propose a robust version, robust in the sense that it is not affected by probability weighting or uncertainty as modelled by biseparable utility.

Definition 4 We call $m$ a robust preference midpoint of $[x, y]$ if Eq. 5 holds for some $r_{1}, r_{2}, x, y$, and $m$ with $r_{1} \leq r_{2} \leq x<y$ or $x<y \leq r_{1} \leq r_{2}$.

Definition 5 We call

$$
\mathcal{A}_{[x, y]}^{r}=\frac{1}{m-x}-\frac{1}{y-m}
$$

a robust discrete Arrow-Pratt index of $[x, y]$ if $m$ is a robust preference midpoint of $[x, y]$.

Definition 6 Robust $\mathcal{A}$-consistency holds if, for each $(x, y) \in \mathbb{R}_{+}^{2}$ with $x<y$, there is at most one $\mathcal{A}_{[x, y]}^{r}$.

As for Theorem 1, the next result generalizes, for two states, Corollary 10 of Köbberling and Wakker (2003) and, therefore, according to these authors' section 7, Ghirardato and Marinacci (2001).

Theorem 2 Under Structural Assumption 1, robust $\mathcal{A}$-consistency is equivalent to biseparable utility.

We have established that the discrete Arrow-Pratt index can be consistently defined for an interval under expected utility, and that the same holds for the robust version under biseparable utility. The discrete Arrow-Pratt index can be directly revealed from preferences, without knowing the decision makers' subjective probabilities. A further advantage of the robust version is that researchers measuring it do not need to know whether the decision makers transform probabilities, have non-additive decision weights, or base their decisions on a set of priors. 
Table 1 Properties of the original Arrow-Pratt indexes preserved by the discrete indexes

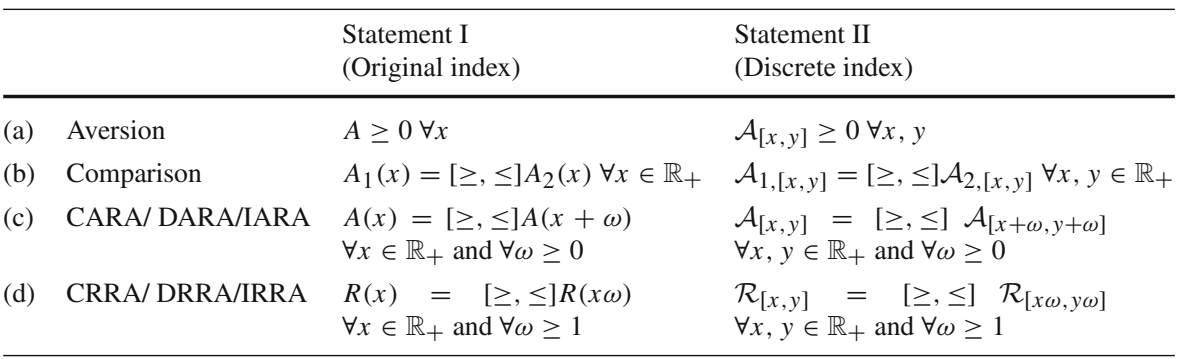

CARA/DARA/IARA mean constant/decreasing/increasing absolute risk aversion. CRRA/DRRA/IRRA mean constant/decreasing/increasing relative risk aversion

\section{Assessing the approximation}

Under expected utility, utility curvature characterizes risk aversion and the ArrowPratt index quantifies this curvature. ${ }^{6}$ For general biseparable utility, utility curvature captures the part of risk and uncertainty attitudes that is attributable to outcomes. The Arrow-Pratt index tells us how risk averse the decision makers would be if they were expected-utility maximizers. In this section, we present the results for expected utility only. The same results hold true if we replace expected utility by biseparable utility and $\mathcal{A}_{[x, y]}$ by $\mathcal{A}_{[x, y]}^{r}$.

The relative version of the Arrow-Pratt index, capturing relative risk aversion under expected utility, is $R(x)=x A(x)$. We similarly define a discrete relative Arrow-Pratt index:

Definition 7 We call

$$
\mathcal{R}_{[x, y]}=m \mathcal{A}_{[x, y]}
$$

a discrete relative Arrow-Pratt index of $[x, y]$ if $m$ is a preference midpoint of $[x, y]$.

The discrete Arrow-Pratt indexes were built by taking linear approximations of derivatives. However, do they preserve the properties of the original indexes? For instance, is shift-invariance of $A(x)$, a.k.a. constant absolute risk aversion (CARA), preserved by our approximation? Table 1 describes important properties of $A(x)$ and $R(x)$, and the corresponding properties of discrete indexes $\mathcal{A}_{[x, y]}$ and $\mathcal{R}_{[x, y]}$. Theorem 3 establishes the equivalence, when utility is smooth (to ensure that $A$ and $R$ exist).

Theorem 3 Assume that $\succsim \succsim_{1}$, and $\succsim_{2}$ maximize expected utility and that the corresponding utility functions are twice continuously differentiable on $\mathbb{R}_{+}$(such that $A$, $A_{1}$, and $A_{2}$ are well-defined on $\left.\mathbb{R}_{+}\right)$. Then statement $I .(i)$ is equivalent to statement II.(i) for all $i \in\{a, b, c, d\}$ in Table 1 .

\footnotetext{
${ }^{6}$ For alternative definitions or measurements of (comparative) risk aversion, see Lajeri and Nielsen (2000) and Eeckhoudt et al. (2017).
} 


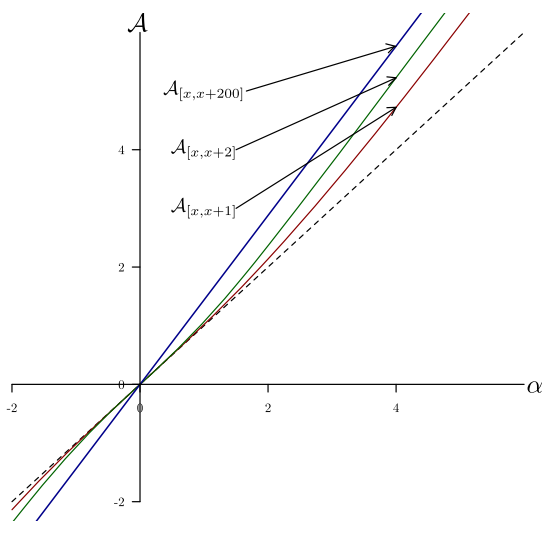

(a) $\mathcal{A}$ as a function of the CARA parameter

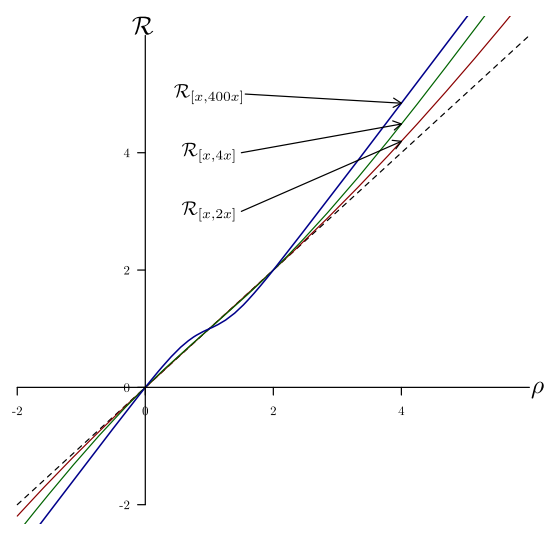

(b) $\mathcal{R}$ as a function of the CRRA parameter

Fig. 2 Discrete indexes for CARA and CRRA utility functions. Note: The CARA function is defined as $u(x)=1-e^{-\alpha x}$ and the CRRA function as $u(x)=\frac{x^{1-\rho}}{1-\rho}$ (and $u(x)=\ln (x)$ if $\left.\rho=1\right)$. The curves represent $\mathcal{A}_{[x, x+\omega]}$ and $\mathcal{R}_{[x, x \omega]}$ as a function of $\alpha$ and $\rho$ respectively. Each curve corresponds to a specific value of $\omega$, for all $x>0$

Theorem 3 establishes that comparative and invariance properties of the original indexes are preserved by the discrete approximations. Absolute and relative risk aversion can therefore be directly tested with the discrete Arrow-Pratt indexes. Hence, Theorem 3 provides an alternative to testing CARA vs. CRRA by fitting functions as often done in the literature (e.g. Meyer 2010; Apesteguia and Ballester 2018).

The discrete and the original indexes share the same properties but how close are they? In consumer behavior analysis, the arc elasticity of demand is close to the (point) elasticity if (i) the degree of convexity of the demand curve is not too extreme and (ii) the interval on which it is measured is not too wide. We would expect a similar result to hold for our discrete indexes with respect to the original ones. Let us proceed with a sort of 'sanity check': if the preferences satisfy CARA or CRRA, i.e., maximize exponential or power utility, how well would our indexes approximate the utility parameter (i.e., the original indexes)? CARA and CRRA functions are very much used in both the theoretical and experimental literature even though they may not best captures utility curvature. In fact, common empirical findings support DARA and IRRA (see Wakker 2010, p. 83). The CARA and CRRA parametric forms are simply convenient tools to check how good the approximations are when the original indexes do not depend on wealth. The same exercise with other utility functions would necessitate to make assumptions about wealth.

Figure 2a is based on $u(x)=1-e^{-\alpha x}$ (giving $A(x)=\alpha$ ) and displays $\mathcal{A}_{[x, x+\omega]}$ as a function of $\alpha$ for three values of $\omega$. Not surprisingly, $\mathcal{A}$ is hardly distinguishable from $\alpha$ between -1.5 and 1.5 and for small intervals $(\omega=1)$. The same holds for $\alpha$ between -1 and 1 and $\omega=2$. The approximation is less precise for larger interval width but, interestingly, the approximation gap does not explode. For extreme curvatures (e.g. $\alpha=5$ ), going from $\omega=1$ to $\omega=2$ has the same impact as going from $\omega=2$ to $\omega=200$. The curve we would obtain for $\omega=10$ is extremely close to that for 
$\omega=200$. Panel b of Fig. 2 replicates this exercise for $u(x)=\frac{x^{1-\rho}}{1-\rho}$ (giving $R(x)=\rho$ ) and $\mathcal{R}$. It plots $\mathcal{R}_{[x, x \omega]}$ as a function of $\rho$ for three values of $\omega$. For any $x>0$ and intervals of size $x$ or even $3 x$ ( $\omega=2$ or 4 respectively), $\mathcal{R}$ almost perfectly matches $\rho$ on $[0,2]$. In fact, for any $\omega, \mathcal{R}_{[x, x \omega]}$ is exactly $\rho$ at 0 (the obvious linear case) but also at 1 (logarithmic utility) and at $2\left(u(x)=-\frac{1}{x}\right){ }^{7}$ We again observe that for extreme curvatures, the approximation does not explode when making the size of the interval much bigger (replacing $\omega=4$ by $\omega=400$ ). Overall, $\mathcal{R}$ is closer to $\rho$ than $\mathcal{A}$ is to $\alpha$.

\section{Elicitation and comparison with alternatives}

The goal of our paper is to provide a method to obtain the Arrow-Pratt index in applied studies and surveys. In many lab and field studies, certainty equivalents $c$ are elicited for bets $(x, y)$, typically with equally likely states (Cohen et al. 1987; Dohmen et al.2011). By definition of certainty equivalents, we have $(c, c) \sim(x, y)$ and both states being equally likely implies $(x, c) \sim(c, x)$. Hence, certainty equivalents of 50-50 bets are special cases of preference midpoints as defined in Eq. 5 (with $c=r_{2}=m$ and $r_{1}=x$ ). We can therefore use $c$ to get the discrete Arrow-Pratt index. However, it does not give the robust discrete Arrow-Pratt index. This approach therefore relies on expected utility and on the possibility to credibly implement equally likely states. Eliciting robust preference midpoints under risk and uncertainty, with no assumption about the decision maker's beliefs, can be done by adapting Wakker and Deneffe's (1996) tradeoff method. In Electronic Supplementary Material, we introduce an algorithm to measure robust midpoints and demonstrate how it can be implemented in an experiment.

Other definitions of preference midpoints have been proposed, e.g. by Vind (1991) and Ghirardato et al. (2003). These definitions have theoretical advantages. For instance, the definition of Vind (1991) allows for state-dependent utility. Statedependency would yield different values of our index for one state of nature than for the other, thereby violating (robust) $\mathcal{A}$-consistency. Vind's approach could help there. The main drawback, however, if that eliciting one midpoint requires four indifferences with his approach (or three for Ghirardato et al. 2003) while the definition used in the present paper requires only two. These indifferences must be obtained simultaneously if one wants to control the interval $[x, y]$, making the elicitation already complex with two indifferences (see Electronic Supplementary Material). Our primary goal being the observability of the Arrow-Pratt index, the fewer indifferences, the better (see Van de Kuilen and Wakker (2011) for a similar argument). By contrast, Ghirardato and Pennesi (2019) make use of the power of Vind's (1991) preference midpoint to characterize ambiguity models that we did not address in this paper, such as Klibanoff et al.'s (2005) smooth ambiguity model. For such models, our (robust) index would not have the same uniqueness property as it has for biseparable utility. Only for the

\footnotetext{
7 If $u(x)=\ln (x)$, the preference midpoint is the geometric midpoint $m=(x y)^{0.5}$. Then $\mathcal{R}_{[x, y]}=(x y)^{0.5}\left[\frac{1}{(x y)^{0.5}-x}-\frac{1}{y-(x y)^{0.5}}\right]=1$. If $u(x)=\frac{-1}{x}, m=\frac{2 x y}{x+y}$ and $\mathcal{R}_{[x, y]}=$ $\frac{2 x y}{x+y}\left[\frac{1}{\frac{2 x y}{x+y}-x}-\frac{1}{y-\frac{2 x y}{x+y}}\right]=2$.
} 
latter model is our robust index purely related to utility curvature and not affected by ambiguity attitudes.

We now turn to comparing the indexes with alternatives. Assuming expected utility, one could also seek to obtain CARA or CRRA parameters from certainty equivalents of 50-50 and use those parameters as indexes of risk aversion. We are not aware of an analytical solution that would give the CARA and CRRA parameter as a function of certainty equivalents in general. The advantage of $\mathcal{A}$ and $\mathcal{R}$ is the ease with which they can be computed, while CARA and CRRA parameters necessitate non-linear fitting.

In the literature, ad hoc measures of risk aversion have often been derived from certainty equivalents. For instance, authors have been using the certainty equivalent divided by $y$ for lotteries of the type $(0, y)$ (Tversky and Kahneman 1992) or the risk premium (certainty equivalent minus expected value) divided by $|y-x|$ (Bouchouicha and Vieider 2017). The properties of such indexes have not always been well understood. Thus, they may depend on stakes in undesirable manners, for instance, converging to risk neutrality for small stakes. Then stake size becomes a confound for statistical inferences. Our indexes avoid such confounds, being theoretically founded and normalizing at the right order of magnitude.

Alternatively, some papers directly report risk premiums or relative risk premiums (e.g. Bruhin et al. 2010; Rieger et al. 2014). Risk premiums include both risk attitudes and the properties (e.g. variance) of the lottery. One could consider dividing the risk premium by half the variance-i.e. reversing the way Pratt (1964) derived the indexbut it might already pose difficulties with small amounts like $\pm \$ 10$. Consider a binary risk, $\pm x$ with equal chance. The maximum risk premium is also $x$ but the variance increases in $x$ in a quadratic way so that the approximation of the Arrow-Pratt index converges towards 0 . For instance, $x=10$ restricts the possible values derived from the approximation to the range $[-0.2,0.2]$ whereas the Arrow-Pratt index can take values between $-\infty$ and $+\infty$. We show in Appendix $C$ that our approach dominates this approach based on risk premiums.

Another approach to obtain the Arrow-Pratt index assuming CARA is to determine $\omega$ such that $x \sim\left(x+\omega, x-\frac{\omega}{2}\right)$ with both states equally likely and $x$ the current wealth. If $u(x)=1-e^{-\alpha x}$, then $\omega \approx \frac{1}{\alpha}$ (Wakker 2010, Observation 3.5.3, p. 82). This approach has been implemented in some major panel surveys, e.g. in Germany (Dohmen et al. 2011) and the US (Barsky et al. 1997). In Appendix C, we demonstrate that this method, developed for one parametric family, can have difficulties for other families and be difficult to implement with monetary incentives.

\section{Conclusion}

The Arrow-Pratt index, cornerstone of the risk and uncertainty literature, is not directly observable because it is based on infinitesimal changes of a subjective, non-directly observable, concept (utility). We proposed a discrete approximation directly revealed from two indifferences and studied its properties. The consistency of our (robust) index is tightly linked to expected utility (biseparable utility). This consistency is a necessary and sufficient condition for expected utility when the state space is binary. 
Our approach is suited whether objective probabilities are available (risk) or not (uncertainty). We showed that it can provide robustness against probability transformations and the existence of multiple priors. This robustness is important for applications, where researchers will want (i) to use naturally occurring events people face everyday to ensure external validity and (ii) to ensure internal validity of the measurement by allowing for deviations from expected utility. Thus, we made the Arrow-Pratt index directly observable and we also made it suited for empirical applications.

Acknowledgements The authors are grateful to Peter Wakker for his numerous insights. The research of A. Baillon was made possible by Vidi grant \#452-13-013 from N.W.O. The research of O. L'Haridon was made possible by AIS-2013 grant from Rennes Metropole and financial support from Institut Universitaire de France, Académie des Sciences and Koninklijke Nederlandse Akamedie van Wetenschappen.

Open Access This article is licensed under a Creative Commons Attribution 4.0 International License, which permits use, sharing, adaptation, distribution and reproduction in any medium or format, as long as you give appropriate credit to the original author(s) and the source, provide a link to the Creative Commons licence, and indicate if changes were made. The images or other third party material in this article are included in the article's Creative Commons licence, unless indicated otherwise in a credit line to the material. If material is not included in the article's Creative Commons licence and your intended use is not permitted by statutory regulation or exceeds the permitted use, you will need to obtain permission directly from the copyright holder. To view a copy of this licence, visit http://creativecommons.org/licenses/by/4.0/.

\section{A Linear approximations}

Here we show how our discrete Arrow-Pratt index is a ratio of linear approximation of the elements of the Arrow-Pratt index at the midpoint $m$, following the approach of Rohde (2019) to define discrete of decreasing impatience. The Taylor series of $u$ on the right and on the left sides of $m$ are:

$$
u(y) \approx u(m)+(y-m) u^{\prime}(m)+\frac{(y-m)^{2}}{2} u^{\prime \prime}(m),
$$

and:

$$
u(x) \approx u(m)-(m-x) u^{\prime}(m)+\frac{(m-x)^{2}}{2} u^{\prime \prime}(m) .
$$

These equations can be rewritten as:

$$
\frac{y-m}{2} u^{\prime \prime}(m) \approx \frac{u(y)-u(m)}{y-m}-u^{\prime}(m),
$$

and:

$$
\frac{m-x}{2} u^{\prime \prime}(m) \approx \frac{u(m)-u(x)}{m-x}+u^{\prime}(m)
$$

Combining Eqs. 12-13 and using $u(y)-u(m)=u(m)-u(x)=\frac{u(y)-u(x)}{2}$ gives:

$$
u^{\prime \prime}(m) \approx \frac{u(y)-u(x)}{(y-m)(y-x)}-\frac{u(y)-u(x)}{(m-x)(y-x)}=\mathcal{C}(x, y) .
$$


This shows how $\mathcal{C}$ is a linear approximation of $u^{\prime \prime}$ at $m$. Restricting Eqs. 10-11 at order 1 and combining them also gives:

$$
u^{\prime}(m) \approx \frac{u(y)-u(x)}{y-x}=\mathcal{S}(x, y) .
$$

In other words, when $x$ and $y$ are close enough, the derivative at $m$ can be approximated by the average slope of $u$ between $x$ and $y$. Dividing Eq. 14 by 15 shows that $\mathcal{A}$ is the ratio of the linear approximation of $u^{\prime \prime}$ and $u^{\prime}$ at $m$.

\section{B Proofs}

\section{B.1 Relating $\mathcal{A}$-consistency to tradeoff consistency and the hexagon condition}

To prepare for the proofs of Theorems 1 and 2 we first establish two lemmas that together relate $\mathcal{A}$-consistency to other properties of the literature. To simplify the proofs of the lemmas, we introduce the following notation: $x_{s} y$ describes the bet $(x, y)$ if $s=s_{1}$ and $(y, x)$ if $s=s_{2}$. Furthermore, two bets $x_{s} y$ and $t_{s} v$ are comonotonic if they assign their higher outcome to the same state (i.e. either $x \geq y$ and $t \geq v$ or $x \leq y$ and $t \leq v$ ).

Definition 8 (Köbberling and Wakker 2003) [Comonotonic] strong indifferencetradeoff consistency holds if for all $x, y, m, n, r, t, v, z \in \mathbb{R}_{+}$and $s, s^{\prime} \in S$, $x_{s} t \sim m_{s} r \& n_{s} t \sim y_{s} r \& x_{s^{\prime}} z \sim m_{s^{\prime}} v \Rightarrow n_{s^{\prime}} z \sim y_{s^{\prime}} v$ [with $x_{s} t, m_{s} r, n_{s} t$, and $y_{s} r$ comonotonic and $x_{s^{\prime}} z, m_{s^{\prime}} v, n_{s^{\prime}} z$, and $y_{s^{\prime}} v$ comonotonic as well].

In their Corollary 10, Köbberling and Wakker (2003) used a slightly different version, called [comonotonic] tradeoff consistency. Their Corollary 28 shows that [comonotonic] strong indifference-tradeoff consistency is equivalent to [comonotonic] tradeoff consistency under the conditions assumed in their Corollary 10 and in our Structural Assumption 1.

Definition 9 (Comonotonic) midpoint consistency holds if for all $x, y, m, r, t, v, z \in$ $\mathbb{R}_{+}$and $s, s^{\prime} \in S, x_{s} t \sim m_{s} r \& m_{s} t \sim y_{s} r \& x_{s^{\prime}} z \sim m_{s^{\prime}} v \Rightarrow m_{s^{\prime}} z \sim y_{s^{\prime}} v$ [with $x_{s} t$, $m_{s} r, m_{s} t$, and $y_{s} r$ comonotonic and $x_{s^{\prime}} z, m_{s^{\prime}} v, m_{s^{\prime}} z$, and $y_{s^{\prime}} v$ comonotonic as well].

Definition 10 The [comonotonic] hexagon condition holds if for all $x, y, m, r, t, z \in$ $\mathbb{R}_{+}$and $s \in S, x_{s} t \sim m_{s} r \& m_{s} t \sim y_{s} r \& x_{s} z \sim m_{s} t \Rightarrow m_{s} z \sim y_{s} t$ [with $x_{s} t, m_{s} r$, $m_{s} t$, and $y_{s} r$ comonotonic and $x_{s} z, m_{s} t, m_{s} z$, and $y_{s} t$ comonotonic as well].

Lemma 2 (Comonotonic) strong indifference-tradeoff consistency $\Rightarrow$ [comonotonic] midpoint consistency $\Rightarrow$ the [comonotonic] hexagon condition.

Proof Midpoint consistency requires the condition for tradeoff consistency only when $n=m$. The hexagon condition requires the condition for midpoint consistency only when $v=t$ and $s^{\prime}=s$. The same arguments apply to the comonotonic conditions. 
Lemma 3 Under Structural Assumption 1, [robust] $\mathcal{A}$-consistency implies [comonotonic] midpoint consistency.

Proof $\mathcal{A}$-consistency is equivalent to requiring that for each $[x, y]$, there is at most one $m$ satisfying Eq. 5 for any $x<y$. By monotonicity, it cannot be $x$ or $y$. We next show that this implies midpoint consistency.

Assume $x_{s} t \sim m_{s} r \& m_{s} t \sim y_{s} r \& x_{s^{\prime}} z \sim m_{s^{\prime}} v$ and $x<y$ so that, by monotonicity, $x<m<y$. We allow $s=s^{\prime}$ or $s \neq s^{\prime}$.

Case 1 Assume, for contradiction, $m_{s^{\prime}} z \succ y_{s^{\prime}} v$, with $x<y$ and, therefore, $x<$ $m<y$ by monotonicity. By continuity, there exists $n$ between $x$ and $m$ such that $n_{s^{\prime}} z \sim y_{s^{\prime}} v$ and by monotonicity $n<m$, and therefore $x_{s^{\prime}} z \succ n_{s^{\prime}} v$. Now consider the indifference curve between $n_{s^{\prime}} z$ and $y_{s^{\prime}} v$, that is, all points $\alpha_{s^{\prime}} \beta$ with $\alpha_{s^{\prime}} \beta \sim y_{s^{\prime}} v$, $n \leq \alpha \leq y$ and $v \leq \beta \leq z$. Let us start from $\alpha=y$ and decrease $\alpha$ incrementally, each time determining the corresponding $\beta$. Up to $\alpha=m$, we know that $\beta$ is such that $x_{s^{\prime}} \beta \precsim \alpha_{s^{\prime}} v$ because $x_{s^{\prime}} \beta \precsim x_{s^{\prime}} z \sim m_{s^{\prime}} v \precsim \alpha_{s^{\prime}} v$ ( $\alpha$ is better than $m$ and $\beta$ is worse than $z$ ). When we reach $\alpha=n$, the preference has reversed and we have $x_{s^{\prime}} \beta \succ \alpha_{s^{\prime}} v$ because $\left(x_{s^{\prime}} z \succ n_{s^{\prime}} v\right)$. Hence, by continuity and connectedness of the aforementioned indifference curve, there exists a pair $\alpha^{*}$ and $\beta^{*}$ such that $x_{s^{\prime}} \beta^{*} \sim \alpha_{s^{\prime}}^{*} v$ and $\alpha_{s^{\prime}}^{*} \beta^{*} \sim y_{s^{\prime}} v$ (by construction). Therefore, both $m$ and $\alpha^{*}$ satisfy a version of Eq. 5 , contradicting $\mathcal{A}$-consistency.

Case 2 Next assume, for contradiction, $m_{s^{\prime}} z \prec y_{s^{\prime}} v$. By monotonicity and continuity, there must exist $y^{\prime}$ with $x<y^{\prime}<y$ such that $m_{s^{\prime}} z \sim y_{s^{\prime}}^{\prime} v \cdot y^{\prime}<y$ further implies that $m_{s} t \succ y_{s}^{\prime} r$. We therefore have $x_{s^{\prime}} z \sim m_{s^{\prime}} v \& m_{s^{\prime}} z \sim y_{s^{\prime}}^{\prime} v \& x_{s} t \sim m_{s} r$ but $m_{s} t \succ y_{s}^{\prime} r$ (with $x<y^{\prime}$ and therefore $x<m<y^{\prime}$ by monotonicity). By the proof of the previous paragraph, $\mathcal{A}$-consistency is violated.

We now turn to robust $\mathcal{A}$-consistency and comonotonic midpoint consistency. First notice that the restriction applied to Eq. 5 in the definition of robust $\mathcal{A}$-consistency implies that the bets must be comonotonic. Second, the proof of the previous two paragraphs preserved the ordering of outcomes in bets and therefore comonotonicity. Especially, $n, \alpha$ and $y^{\prime}$ are between $x$ and $y$ and $\beta$ between $v$ and $z$. Hence, the previous two paragraphs also prove that a violation of comonotonic midpoint consistency implies the existence of two midpoints (obtained in a comonotonic way) and therefore a violation of robust $\mathcal{A}$-consistency.

\section{B.2 Existence of preference midpoints}

Lemma 4 Under Structural Assumption 1, there exists $(x, y) \in \mathbb{R}_{+}^{2}$ with $x \neq y$ such that there exists a robust preference midpoint, and therefore a preference midpoint as well.

Proof Structural Assumption 1 implies that there exists a continuous monotonic function $V$ representing $\succsim$. We can get $\left(x, r_{2}\right)$ and $\left(y, r_{1}\right)$ such that $V\left(x, r_{2}\right)=V\left(y, r_{1}\right)$ and either $r_{1}<r_{2} \leq x<y$ or $x<y \leq r_{1}<r_{2}$. For any $m \in[x, y]$, we define $r(m)$ such that $V(x, r(m))=V\left(m, r_{1}\right)$. By the Structural Assumption, $r(m)$ exists and $r_{1} \leq r(m) \leq r_{2}$. 
The function $V(m, r(m))-V(y, r 1)$ is continuous in $m$, positive at $m=y$ (because $r(y)=r_{2}>r_{1}$ ), and negative at $m=x$ (because $r(x)=r_{1}$ and $x<y$ ). By the intermediate value property, it is 0 at some $m$. This $m$ is the robust preference midpoint.

Lemma 5 Under expected utility, for all $(x, y) \in \mathbb{R}_{+}^{2}$, there exists a preference midpoint.

Proof Take any $x$ and $y$, with $y \geq x$ and assume, without loss of generality, that $p_{1} \leq 1 / 2$. Continuity of utility implies that there is $m$ such that $u(m)=\frac{u(x)+u(y)}{2}$. Fix $r_{1}=0$ and we can normalize $u$ such that $u(0)=0$. Let $r_{2}$ be defined by $u\left(r_{2}\right)=$ $\frac{p_{1}}{1-p_{1}}(u(y)-u(m))$. It must exist by continuity because $u\left(r_{1}\right) \leq u\left(r_{2}\right) \leq u(y)$. Eq. 5 holds.

Observation 1 Under Structural Assumption 1, there may exist $(x, y) \in \mathbb{R}_{+}^{2}$ such that no robust preference midpoint exists.

Proof Assume biseparable preferences. Take $x=0$ and $y=1$. Assume $u(0)=0$, $u(1)=1, \pi_{1}=\pi_{2}=0.375$ and that $u$ is bounded by 1.1 . There are no $r_{1}$ and $r_{2}$ higher than $y$ that would satisfy $u\left(r_{2}\right)-u\left(r_{1}\right)=\frac{1-\pi_{i}}{\pi_{i}} \frac{u(y)-u(x)}{2}=0.3$, which would be necessary for Eq. 5 to hold.

\section{B.3 Proof of Theorem 1}

Proof Step 1: Expected utility implies $\mathcal{A}$-consistency Assume expected utility. The function $u$ is strictly increasing and continuous and therefore, there is only one $m$ such that $u(m)=\frac{u(x)+u(y)}{2}$, which, by Lemma 1, implies that there can be at most one preference midpoint. Hence $\mathcal{A}$-consistency holds.

Step 2: $\mathcal{A}$-consistency implies an additive representation: By Lemmas 2 and 3, we have the hexagon condition. Theorem III.4.1 of Wakker (1989) implies that there exist two continuous functions $V_{1}$ and $V_{2}$, unique up to locations and a joint unit, such that preferences can be represented by $V\left(x_{1}, x_{2}\right)=V_{1}\left(x_{1}\right)+V_{2}\left(x_{2}\right)$ because our monotonicity condition implies Wakker's coordinate independence and also implies that both states are essential.

Step 3: Showing that $V_{1}$ is an affine transformation of $V_{2}$ : By monotonicity, $V_{1}$ and $V_{2}$ must be strictly increasing on top of being continuous. Hence there exists a strictly increasing function $\phi$ such that $V_{1}=\phi \circ V_{2}$. By continuity, for all $z \in$ $\mathbb{R}_{+}$, we can find a neighborhood $I \subset \mathbb{R}_{+}$around $z$ where all pairs $x$ and $y$ in $I$ have preference midpoints that can be observed both on the first state and on the second state; i.e., for all pairs $x$ and $y$ we can find $m_{1}, t_{1}, r_{1}, m_{2}, t_{2}, r_{2}$ such that $\left(x, t_{2}\right) \sim\left(m_{1}, r_{2}\right),\left(m_{1}, t_{2}\right) \sim\left(y, r_{2}\right),\left(t_{1}, x\right) \sim\left(r_{1}, m_{2}\right)$ and $\left(t_{1}, m_{2}\right) \sim\left(r_{1}, y\right)$. By midpoint consistency, $m_{1}=m_{2}$. Midpoints satisfy $V_{i}\left(m_{i}\right)=\frac{V_{i}(x)+V_{i}(y)}{2}$ and, hence, $\phi\left(\frac{V_{1}(x)+V_{1}(y)}{2}\right)=\frac{\phi\left(V_{1}(x)\right)+\phi\left(V_{1}(y)\right)}{2}$. Hence $\phi$ is affine on $I$ (implied by Observation 89 of Hardy et al. 1934). To sum up, for all $z$, we can find a neighborhood $I$ where $\phi$ is affine and, hence, has second derivative 0 . Affinity holds globally.

Step 4: Defining $p_{1}: V_{1}$ and $V_{2}$ can be rescaled such that they have the same value 0 at 0 and thus, such that $\phi$ is linear. Hence, there exists $p_{1}$ such that $V_{1}(x)=\frac{1-p_{1}}{p_{1}} V_{2}(x)$. 
Step 5: Defining $u: V_{1}$ and $V_{2}$ are continuous and strictly increasing. Consequently, $0<p_{1}<1$ and we can define $u=\frac{V_{1}}{p_{1}}$, with $u$ also continuous and strictly increasing.

\section{B.4 Proof of Theorem 2}

Proof Necessity of robust $\mathcal{A}$-consistency is proven as done for expected utility.

We now show that robust $\mathcal{A}$-consistency implies biseparable utility. Let us first define the following comonotonic sets of bets: $\mathbb{R}_{+}^{2} \downarrow=\left\{(x, y) \in \mathbb{R}_{+}^{2}: x \geq y\right\}$ and $\mathbb{R}_{+}^{2} \uparrow=\left\{(x, y) \in \mathbb{R}_{+}^{2}: x \leq y\right\}$.

Step 1: Obtaining expected utility on $\mathbb{R}_{+}^{2} \downarrow$ : We first consider the interior of $\mathbb{R}_{+}^{2} \downarrow$. Under Structural Assumption 1, robust $\mathcal{A}$-consistency implies the comonotonic hexagon condition by Lemmas 2 and 3 . The comonotonic hexagon condition implies the hexagon condition restricted to $\operatorname{int}\left(\mathbb{R}_{+}^{2} \downarrow\right)$. By Corollary 2.3 of Chateauneuf and Wakker (1993), there exists an additive representation $V\left(x_{1}, x_{2}\right)=V_{1}\left(x_{1}\right)+V_{2}\left(x_{2}\right)$ in $\operatorname{int}\left(\mathbb{R}_{+}^{2} \downarrow\right)$ because $\operatorname{int}\left(\mathbb{R}_{+}^{2} \downarrow\right)$ is a so-called comoncone and therefore satisfies Assumption 2.1 of Chateauneuf and Wakker (1993) as shown in their Lemma C.3. As we did in the proof of Theorem 1, we can further use midpoint consistency on $\mathbb{R}_{+}^{2} \downarrow$ to show that $V_{1}$ is a linear transformation of $V_{2}$. We therefore obtain a representation $\pi_{1} u\left(x_{1}\right)+\left(1-\pi_{1}\right) u\left(x_{2}\right)$ on $\operatorname{int}\left(\mathbb{R}_{+}^{2} \downarrow\right)$.

Step 2: Obtaining expected utility on $\mathbb{R}_{+}^{2} \uparrow$ : The exact same steps applied to $\operatorname{int}\left(\mathbb{R}_{+}^{2} \uparrow\right)$ give a representation $\left(1-\pi_{2}\right) v\left(x_{1}\right)+\pi_{2} v\left(x_{2}\right)$.

Step 3: Relating $u$ and $v$ : comonotonic midpoint consistency implies that $u$ and $v$ are cardinal transformation of each other.We can therefore use the same one, $u$.

Step 4: Obtaining a representation on $\mathbb{R}_{+}^{2}$ : Following Step 3 and by continuity, we obtain a representation Eq. 7 on both $\operatorname{int}\left(\mathbb{R}_{+}^{2} \downarrow\right)$ and $\operatorname{int}\left(\mathbb{R}_{+}^{2} \uparrow\right)$. Because both comonotonic sets contain the constant acts and every bet has an equivalent constant bet, the representation holds on the whole domain $\mathbb{R}_{+}^{2}$ by transitivity.

The sufficiency part in the proofs of Theorems 1 and 2 shows that we only use (comonotonic) midpoint consistency, which justifies our generalization claims.

\section{B.5 Proof of Theorem 3}

Proof Assume that $\succsim_{1}$ and $\succsim_{2}$ maximize expected utility. Theorem 2.3 of Baillon et al. (2012) established that $\succsim_{2}$ having lower preference midpoints than $\succsim_{1}$ is equivalent to $u_{2}$ being more concave than $u_{1}$. [Their proof could be directly adapted to biseparable utility.] Furthermore, it is well-known that $u_{2}$ being more concave than $u_{1}$ is equivalent to Statement I.(b). Having lower preference midpoints is equivalent to a larger discrete Arrow-Pratt index. This proves I.(b) $\Leftrightarrow$ II.(b).

Applying this result with $u_{2}$ linear, $u_{2}(x)=u_{1}(x+\omega)$, and $u_{2}(x)=u_{1}(x \omega)$ establishes the equivalence between I and II in rows (a), (c) and (d) respectively. 
Table 2 Approximations with a risk premium

\begin{tabular}{|c|c|c|c|c|c|c|c|c|}
\hline \multirow[b]{2}{*}{$\alpha$} & \multicolumn{2}{|l|}{$\omega=0.1$} & \multicolumn{2}{|l|}{$\omega=1$} & \multicolumn{2}{|l|}{$\omega=2$} & \multicolumn{2}{|l|}{$\omega=200$} \\
\hline & $\mathcal{A}_{[x, x+0.1]}$ & $\tilde{A}_{[x, x+0.1]}$ & $\mathcal{A}_{[x, x+1]}$ & $\tilde{A}_{[x, x+1]}$ & $\mathcal{A}_{[x, x+2]}$ & $\tilde{A}_{[x, x+2]}$ & $\mathcal{A}_{[x, x+200]}$ & $\tilde{A}_{[x, x+200]}$ \\
\hline-2.00 & -2.00 & -2.00 & -2.14 & -1.74 & -2.36 & -1.33 & -2.88 & -0.02 \\
\hline-1.50 & -1.50 & -1.50 & -1.56 & -1.38 & -1.69 & -1.14 & -2.16 & -0.02 \\
\hline-1.00 & -1.00 & -1.00 & -1.02 & -0.96 & -1.07 & -0.87 & -1.44 & -0.02 \\
\hline-0.50 & -0.50 & -0.50 & -0.50 & -0.49 & -0.51 & -0.48 & -0.72 & -0.02 \\
\hline 0.00 & 0.00 & 0.00 & 0.00 & 0.00 & 0.00 & 0.00 & 0.00 & 0.00 \\
\hline 0.50 & 0.50 & 0.50 & 0.50 & 0.49 & 0.51 & 0.48 & 0.72 & 0.02 \\
\hline 1.00 & 1.00 & 1.00 & 1.02 & 0.96 & 1.07 & 0.87 & 1.44 & 0.02 \\
\hline 1.50 & 1.50 & 1.50 & 1.56 & 1.38 & 1.69 & 1.14 & 2.16 & 0.02 \\
\hline 2.00 & 2.00 & 2.00 & 2.14 & 1.74 & 2.36 & 1.33 & 2.88 & 0.02 \\
\hline 2.50 & 2.50 & 2.49 & 2.74 & 2.03 & 3.06 & 1.45 & 3.60 & 0.02 \\
\hline 3.00 & 3.01 & 2.99 & 3.38 & 2.28 & 3.78 & 1.54 & 4.32 & 0.02 \\
\hline 3.50 & 3.51 & 3.48 & 4.04 & 2.48 & 4.50 & 1.60 & 5.04 & 0.02 \\
\hline 4.00 & 4.01 & 3.97 & 4.72 & 2.65 & 5.23 & 1.65 & 5.77 & 0.02 \\
\hline 4.50 & 4.52 & 4.46 & 5.42 & 2.79 & 5.95 & 1.69 & 6.49 & 0.02 \\
\hline 5.00 & 5.03 & 4.95 & 6.12 & 2.90 & 6.68 & 1.72 & 7.21 & 0.02 \\
\hline 5.50 & 5.53 & 5.43 & 6.84 & 3.00 & 7.40 & 1.75 & 7.93 & 0.02 \\
\hline 6.00 & 6.04 & 5.91 & 7.56 & 3.08 & 8.13 & 1.77 & 8.65 & 0.02 \\
\hline 6.50 & 6.56 & 6.39 & 8.28 & 3.15 & 8.85 & 1.79 & 9.37 & 0.02 \\
\hline 7.00 & 7.07 & 6.86 & 9.00 & 3.21 & 9.57 & 1.80 & 10.09 & 0.02 \\
\hline
\end{tabular}

\section{Alternatives and their limitations}

\section{C.1 Approximating the Arrow-Pratt index with a 50-50 bet $\left(w,-\frac{w}{2}\right)$}

Let $x$ be the current wealth of a decision maker. An approach to obtain the Arrow-Pratt index assuming CARA is to determine $\omega$ such that $x \sim\left(x+\omega, x-\frac{\omega}{2}\right)$ with both states equally likely. If $u(x)=1-e^{-\alpha x}$, then $\omega=\frac{2 \ln (0.5+\sqrt{1.25})}{\alpha} \approx \frac{1}{\alpha}$ (Wakker 2010, Observation 3.5.3, p. 82). This can be seeing as fitting an exponential function on one (carefully-chosen) indifference. A first drawback is that it only works for strict risk aversion. Risk neutral and risk seeking decision makers would have $\omega=+\infty$.

To illustrate that a method developed for a parametric family can have difficulties for other families, let us now assume power utility. It can be shown that anyone with a utility less concave than $u(z)=z^{\frac{\ln (2)}{\ln (3)}}$ (which means a relative Arrow-Pratt index smaller than 0.369 ) would pick $\omega>2 x$, taking the risk to lose more than his initial wealth. ${ }^{8}$ It would then be complicated to use such an approach with real monetary incentives in a survey or an experiment.

$\overline{8 \omega>2 x \text { gives } \frac{1}{2}(3 x)^{1-\rho}>x^{1}}-\rho \Rightarrow 3^{1-\rho}>2 \Rightarrow \rho<1-\frac{\ln (2)}{\ln (3)}$. 


\section{C.2 Approximating the Arrow-Pratt index with a risk premium}

We assume $u(x)=1-e^{-\alpha x}$ and approximate the Arrow-Pratt index using the risk premium of a bet that gives $x$ and $x+\omega$ with equal chance. Under expected utility, the preference midpoint is $m$ and corresponds to the certainty equivalent of the bet. This bet has a variance of $\left(\frac{\omega}{2}\right)^{2}$ and the risk premium is $\frac{2 x+\omega}{2}-m$. Dividing the risk premium by half the variance gives the following approximation of the Arrow-Pratt index:

$$
\tilde{A}_{[x, x+\omega]}=\frac{8 x+4 \omega-8 m}{\omega^{2}} .
$$

By definition $m$ must be in $[x, x+\omega]$, which implies $\lim _{\omega \rightarrow+\infty} \tilde{A}_{[x, x+\omega]}=0$. Table 2 displays $\tilde{A}$ for various values of $\omega$ (columns) and $\alpha$ (rows). Table 2 shows the comparison between $\tilde{A}$ and $\mathcal{A}$. For very small risk $(\omega=0.1)$ both are very accurate but any small increase of $\omega$ deteriorates the quality of the approximation using risk premia. In the most extreme case $(\omega=200), \tilde{A}$ completely fails because the large variance makes it inadequate to rely on second-order Taylor approximations. In Table 2, the absolute difference between $\alpha$ and $\mathcal{A}$ is always smaller than between $\alpha$ and $\tilde{A}$.

\section{References}

Abdellaoui, M., Bleichrodt, H., Paraschiv, C.: Loss aversion under prospect theory: a parameter-free measurement. Manag. Sci. 53(10), 1659-1674 (2007)

Allais, M.: Le comportement de l'homme rationnel devant le risque: Critique des postulats et axiomes de l'école americaine. Econometrica 21(4), 503-546 (1953)

Apesteguia, J., Ballester, M.A.: Monotone stochastic choice models: the case of risk and time preferences. J. Politi. Econ. 126(1), 74-106 (2018)

Arrow, K.J.: Aspects of the Theory of Risk-Bearing. Yrjö Jahnssonin Säätiö, Helsinki (1965)

Baillon, A., Driesen, B., Wakker, P.P.: Relative concave utility for risk and ambiguity. Games Econ. Behav. 75(2), 481-489 (2012)

Barsky, R.B., Juster, F.T., Kimball, M.S., Shapiro, M.D.: Preference parameters and behavioral heterogeneity: an experimental approach in the health and retirement study. Q. J. Econ. 112(2), 537-579 (1997)

Bouchouicha, R., Vieider, F.M.: Accommodating stake effects under prospect theory. J. Risk Uncertainty 55(1), 1-28 (2017)

Bruhin, A., Fehr-Duda, H., Epper, T.: Risk and rationality: uncovering heterogeneity in probability distortion. Econometrica 78(4), 1375-1412 (2010)

Chateauneuf, A., Wakker, P.: From local to global additive representation. J. Math. Econ. 22(6), 523-545 (1993)

Cohen, M., Jaffray, J.-Y., Said, T.: Experimental comparison of individual behavior under risk and under uncertainty for gains and for losses. Organ. Behav. Hum. Decis. Process. 39(1), 1-22 (1987)

de Finetti, B.: Sulla preferibilitá. Giornale degli Economisti e Annali di Economia 11(11/12), 685-709 (1952)

Dean, M., Ortoleva, P.: Allais, Ellsberg, and preferences for hedging. Theor. Econ. 12(1), 377-424 (2017)

Dohmen, T., Falk, A., Huffman, D., Sunde, U., Schupp, J., Wagner, G.G.: Individual risk attitudes: measurement, determinants, and behavioral consequences. J. Eur. Econ. Assoc. 9(3), 522-550 (2011)

Eeckhoudt, L., Liu, L., Meyer, J.: Restricted increases in risk aversion and their application. Econ. Theor. 64(1), 161-181 (2017)

Ellsberg, D.: Risk, ambiguity, and the savage axioms. Q. J. Econ. 75(4), 643-669 (1961) 
Ghirardato, P., Marinacci, M.: Risk, ambiguity, and the separation of utility and beliefs. Math. Oper. Res. 26(4), 864-890 (2001)

Ghirardato, P., Pennesi, D.: Mixing without randomness. Working Paper, Collegio Carlo Alberto, University of Torino (2019)

Ghirardato, P., Maccheroni, F., Marinacci, M., Siniscalchi, M.: A subjective spin on roulette wheels. Econometrica 71(6), 1897-1908 (2003)

Ghirardato, P., Maccheroni, F., Marinacci, M.: Differentiating ambiguity and ambiguity attitude. J. Econ. Theory 118(2), 133-173 (2004)

Gilboa, I., Schmeidler, D.: Maxmin expected utility with non-unique prior. J. Math. Econ. 18(2), 141-153 (1989)

Gul, F., et al.: Savage's theorem with a finite number of states. J. Econ. Theory 57(1), 99-110 (1992)

Hardy, G., Littlewood, J., Pólya, G.: Inequalities. Cambridge University Press, Cambridge (1934)

Klibanoff, P., Marinacci, M., Mukerji, S.: A smooth model of decision making under ambiguity. Econometrica 73(6), 1849-1892 (2005)

Köbberling, V., Wakker, P.P.: Preference foundations for nonexpected utility: a generalized and simplified technique. Math. Oper. Res. 28(3), 395-423 (2003)

Lajeri, F., Nielsen, L.T.: Parametric characterizations of risk aversion and prudence. Econ. Theor. 15(2), 469-476 (2000)

Luce, R.D.: Rank-and sign-dependent linear utility models for binary gambles. J. Econ. Theory 53(1), 75-100 (1991)

Machina, M.J.: "Expected utility" analysis without the independence axiom. Econometrica 50(2), 277-323 (1982)

Meyer, J.: Representing risk preferences in expected utility based decision models. Ann. Oper. Res. 176(1), 179-190 (2010)

Montesano, A.: De Finetti and the Arrow-Pratt measure of risk aversion. In: Gavalotti, M. (ed.) Bruno de Finetti Radical Probabilist. College Publications, London (2009)

Pratt, J.W.: Risk aversion in the small and in the large. Econometrica 32(1), 122-136 (1964)

Prelec, D.: Decreasing impatience: a criterion for non-stationary time preference and "hyperbolic" discounting. Scand. J. Econ. 106(3), 511-532 (2004)

Quiggin, J.: Risk perception and risk aversion among Australian farmers. Aust. J. Agric. Econ. 25(2), 160-169 (1981)

Rieger, M.O., Wang, M., Hens, T.: Risk preferences around the world. Manag. Sci. 61(3), 637-648 (2014)

Rohde, K.I.: Measuring decreasing and increasing impatience. Manag. Sci. 65(4), 1700-1716 (2019)

Savage, L.J.: The Foundations of Statistics. Wiley, New York (1954)

Schmeidler, D.: Subjective probability and expected utility without additivity. Econometrica 57(3), 571-587 (1989)

Tversky, A., Kahneman, D.: Advances in prospect theory: cumulative representation of uncertainty. J. Risk Uncertainty 5(4), 297-323 (1992)

Van de Kuilen, G., Wakker, P.P.: The midweight method to measure attitudes toward risk and ambiguity. Manag. Sci. 57(3), 582-598 (2011)

Vind, K.: Independent preferences. J. Math. Econ. 20(1), 119-135 (1991)

Wakker, P.P.: Additive Representations of Preferences: A New Foundation of Decision Analysis. Kluwer Academic Publishers, Dordrecht (1989)

Wakker, P.P.: Prospect Theory: For Risk and Ambiguity. Cambridge University Press, Cambridge (2010)

Wakker, P., Deneffe, D.: Eliciting von Neumann-Morgenstern utilities when probabilities are distorted or unknown. Manag. Sci. 42(8), 1131-1150 (1996)

Werner, K.M., Zank, H.: A revealed reference point for prospect theory. Econ. Theor. 67(4), 731-773 (2019)

Publisher's Note Springer Nature remains neutral with regard to jurisdictional claims in published maps and institutional affiliations. 